\title{
Light scattering of water under magnetic field
}

\author{
Kovalenko V.F., Bordyuk A.Yu. and Shutov S.V. \\ Kherson National Technical University, 24 Berislav Highway, \\ 73008 Kherson, Ukraine, e-mail: shutov_sv@mail.ru
}

Received: 02.10.2009

\begin{abstract}
Effect of static magnetic field with the strength up to $6.4 \times 10^{4} \mathrm{~A} / \mathrm{m}$ on the structural properties of water is investigated at $300 \mathrm{~K}$, using measurements of angular dependences of the scattered laser radiation intensity. This effect manifests itself in the changes of basic structural properties of water such as a set of clusters available, their concentration and sizes. A degree and character of the changes depend on the modes of field influence (e.g., fixed or rotating field), the field strength and action time, the initial set and concentration of clusters in the water, as well as parameters of the field rotation. The structural properties change due to work of the field upon the orientation, along the field direction, of magnetic moments of clusters' elements (hexagonal rings and planes) and the clusters as a whole. This work is spent, on the one hand, on breaking hydrogen bonds that bind the cluster elements, and surmounting a friction force appearing when the clusters rotate in the magnetic field. Besides, it is spent on forming new clusters from the fragments of destroyed ones and forming magnetically ordered clusters from the intact ones.
\end{abstract}

Keywords: light scattering, water clusters, magnetic field.

PACS: 42.62.-b, 61.20.-p, 61.25.-f, 78.35+c, 78.40.Dw

UDC: $591.044 ; 577.3$

\section{Introduction}

It is known (see, e.g., [1-3]) that water exposed to a constant magnetic field changes its physicochemical properties, resulting in increasing rate of different chemical processes and crystallisation of dissolved substances, intensified adsorption processes, and enhanced coagulation and precipitation of impurities. Moreover, the water becomes biologically active. Owing to these properties, magnetic water is widely applied in industry, agriculture, medicine, etc. In spite of those practical applications, physical reasons for the changes in the properties of water occurring under magnetic fields have not yet been ascertained. There are some versions of the explanation. According to one of them, the magnetic field affects the structural properties of water, leading to breakage or distortion of hydrogen bonds of clusters and so to changes in different physicochemical properties. However this explanation still lacks any experimental confirmations. Apparently, this has resulted in sometimes contrary opinions on the structural properties of water under magnetic field. According to [4], the water becomes more structured if compare with the initial one, while the work [5] has predicted that it becomes non-structured. 
Some possibilities for studying the structural properties of water using measurements of its light scattering have been demonstrated in our recent work [6]. The present study deals with the experimental data concerned with the influence of magnetic field on the structural properties of water. The data have been obtained on the basis of angular dependences of the scattered light intensity (so-called scattering indicatrices $I(\Theta)$, with $\Theta$ being the scattering angle). Our results prove the assumption that the magnetic field imposes notable changes in the structural properties of water.

\section{Experimental}

Magnetic field was produced by two identical rod-shaped permanent magnets having the diameter of $5 \mathrm{~mm}$ and the length of $25 \mathrm{~mm}$. They were fixed in a special holder in line in a horizontal plane at the height of laser beam, with the opposite poles aligned towards each other. A homogeneous magnetic field was formed in between the poles. Just that volume of water was affected by the field, which a beam with the diameter of $3 \mathrm{~mm}$ passed through. A design of the holder enabled changing the distance between the butt ends of our magnets and thus regulating the field strength $\vec{H}$. The maximal $\vec{H}$ value reached $\left(\vec{H}_{\max }=6.4 \times 10^{4} \mathrm{~A} / \mathrm{m}\right)$ was determined by the minimal distance between the poles which, in its turn, was set by the external diameter $(10 \mathrm{~mm})$ of a cuvette containing a water probe and disposed between the poles. The $\vec{H}$ magnitudes were measured with a Hall sensor.

Scattering indicatrices of water were measured at the temperature $T=300 \mathrm{~K}$ before (the parameter $\left.I(\Theta)_{\mathrm{b}}\right)$ and right after $\left(I(\Theta)_{\mathrm{a}}\right)$ the field was applied in the horizontal or vertical planes. In the latter case one of the magnets was located vertically under the glass cuvette so that the magnet's top touched bottom of the cuvette. The second magnet on a hanger was descended into the cuvette, so that the distance between its bottom and the top the first magnet provided a required $\overrightarrow{\mathrm{H}}$ value, and the volume of water exposed to the field was levelled with the laser beam. Our apparatus for measuring scattering indicatrices, a measuring technique itself and data processing procedures had been described in detail in the work [6].

The influence of magnetic field was studied using water probes taken from a spring of Vladimirsky Cathedral (Kiev city, Ukraine). In order to determine the shape of water clusters, samples of different origins were also used. These were water probes from a local artesian well (referred to as "spring water"), distilled water and tap one.

\section{Results and discussion}

\subsection{The influence of static magnetic field}

In the majority of our experiments the initial water is characterised by sufficiently smooth scattering indicatrix and by a set of clusters (conditionally referred to as a "typical" one), of which radii $\mathrm{r}$ are equal to $\sim 1.50 \div 1.60 \mu \mathrm{m}, 0.95 \div 1.22 \mu \mathrm{m}, \sim 0.56 \div 0.59 \mu \mathrm{m}, \sim 0.47 \mu \mathrm{m}$, $\sim 0.33 \div 0.36 \mu \mathrm{m}, \sim 0.28 \div 0.30 \mu \mathrm{m}, \sim 0.25 \div 0.26 \mu \mathrm{m}$, and $\sim 0.22 \mu \mathrm{m}$. The radii have been determined following from the shape of dependences

$$
\Theta^{2} I(\Theta)=f(\Theta)
$$

Ukr. J. Phys. Opt. 2010, V11, №1 
and calculated by means of the method considered in [6] ${ }^{*}$ (see Fig. 1).

The influence of static magnetic field parallel to the incident laser beam on the light scattering characteristics manifests itself in changing both the integrated intensity $I_{\Sigma}$ of scattered light and the intensity of different portions of the scattering indicatrices $I(\Theta)_{\text {a }}$. The increasing time $t_{a}$ for which the magnetic field is applied causes non-monotonous decrease in $I_{\Sigma}\left(10-12 \%\right.$ at $\left.t_{\mathrm{a}}=60 \mathrm{~min}\right)$, though the effect is practically independent of the magnetic field strength. Changes in the intensity of different areas of the scattering indicatrices have a multidirectional character $\left(I(\Theta)_{\mathrm{a}}>I(\Theta)_{\mathrm{b}}\right.$ or $\left.I(\Theta)_{\mathrm{a}}<I(\Theta)_{\mathrm{b}}\right)$ and depend on both the $t_{\mathrm{a}}$ and $\overrightarrow{\mathrm{H}}$ parameters.

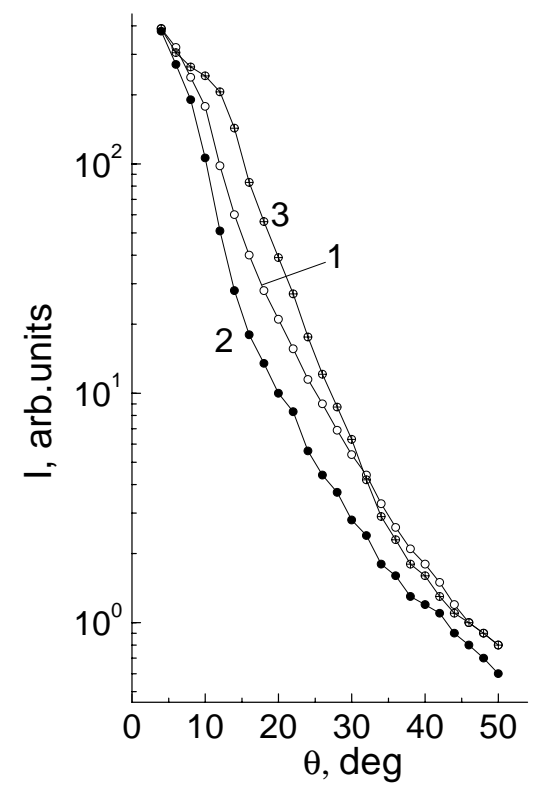

a

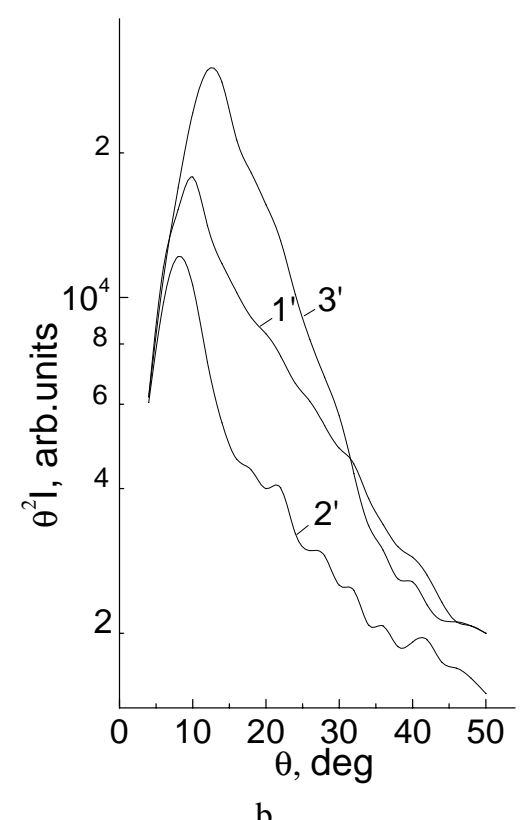

b

Fig. 1. Scattering indicatrices (a) and the corresponding calculated dependences $\Theta^{2} I=f(\Theta)$ (b) for the initial water with "typical" set of clusters (curves 1 and $1^{\prime}$ ), the water containing super-large clusters (curves 2 and 2 ') and the water differing by increased content of middle-sized clusters (curves 3 and $3^{\prime}$ ).

According to [6], under the assumption of single scattering we have

$$
I_{\Sigma}=\sum_{i} \beta N_{i} r_{i} I_{0} V
$$

where $\beta$ denotes a coefficient taking into account the angular aperture of photodetector and its spectral sensitivity, $N_{\mathrm{i}}$ and $r_{\mathrm{i}}$ are respectively the concentration and the radius of clusters of $i$-type, $I_{0}$ the intensity of the incident light, $V$ the volume of the irradiated liquid, and $i$ the number of cluster type or size. It can be seen from Eq. (2) that the $I_{\Sigma}$ change could be conditioned in general by varying size $r_{i}$ of clusters and/or their concentration $N_{i}$. In the conditions of our experiments, the sizes of clusters after the exposure to mag-

\footnotetext{
* Due to natural structuring, the set of clusters mentioned could slightly change with time in the process of our studies, varying in sizes and amount of cluster types.
} 
netic field do not change in practice, however some of the middle and small clusters $(r<0.9 \mu \mathrm{m})$ observed in the initial water do not show up after the exposure. This circumstance allows concluding that decrease of $I_{\Sigma}$ is caused by decreasing concentration of clusters of different types.

Different characters of the intensity changes occurring in separate areas of the scattering indicatrices and different magnitudes of these changes imposed by the field testify to different degrees of increase or decrease in the concentrations of various types of clusters. The most complete information on these and other aspects of magnetic field effect on the structural properties of water might be derived when plotting, for every case of influence, a relative scattering indicatrix $R(\Theta)$, which represents the ratio of scattering indicatrices for a given test measured after and before the influence:

$$
R(\Theta)=\frac{I(\Theta)_{a}}{I(\Theta)_{b}} .
$$

Information value of the $R(\Theta)$ parameter consists in the following. As seen from comparison of curves 1 and 4 in Fig. 2b, we have strictly determined a correlation between the angular positions of extremes of the relative scattering indicatrix and the maxima of the calculated dependence given by Eq. (1). On the basis of this correlation, it is possible to determine the size of clusters, existing in water after the field influence, issuing from the positions of $R(\Theta)$ extremes. The important advantage of this technique for determining sizes is higher resolution for the extremes $R(\Theta)$, as compared with that for the maxima of the curve (1). This enables detecting even clusters invisible in the curve given by Eq. (1) due to their low concentration (e.g., those marked in Fig. $2 b$ by arrows with asterisks).

A better resolution peculiar to the relative scattering indicatrix also makes it easier to reveal super-large clusters formed during the field action, while tracking a presence of periodic fluctuations of the scattered light intensity in the angular interval $\Theta>20^{\circ}$. At their low concentrations, these clusters manifest themselves extremely feebly and inexpressively on both the indicatrices and the curves (1), or even become invisible at all (see curves 2 and curve 2' in Fig. 1 and curves 1 and 4 in Fig. 2b). This advantage allows obtaining more reliable data on the ensemble of scattering clusters.

Besides, utilisation of the relative scattering indicatrix gives a possibility for determining simultaneously both a character and degree of changes in the cluster concentrations caused by the field influence, following from the numerical $R(\Theta)$ values. So, the conditions $R(\Theta)>1$ and $R(\Theta)<1$ mean respectively growing and lowering concentra-

\footnotetext{
* It is shown in the study [7] that in the presence in water of clusters having the sizes that satisfy the condition $\pi r / \lambda \geq 5$, a part of incident radiation passes through them and, after refracting, interferes with the rays diffracted at the same clusters in the angular interval of $20^{\circ}<\Theta \leq 50^{\circ}$, as well as with those reflected from the clusters' surfaces in the interval of $50^{\circ}<\Theta \leq 150^{\circ}$. This interference manifests itself on the scattering indicatrices and the corresponding curves (1) as periodic fluctuations of the scattered intensity in the corresponding angular intervals, of which period facilitates deriving the size of these clusters.
}

Ukr. J. Phys. Opt. 2010, V11, №1 
tions of clusters that form the scattered intensity in a given angular sector. Thus, the $R(\Theta)$ number reflects precisely the portion or percentage of the concentration changes.

In Fig. 2a and Fig. 2b we present the relative scattering indicatrices of water tests having "typical" initial set of clusters for various time exposures $t_{\mathrm{a}}$ and strengths $\vec{H}$ of the static magnetic field. From Fig. 2a it follows that the action of weak fields during 10 min has a selective character. Namely, in the small-angle area $\left(\Theta \leq 15^{\circ}\right)$ we have

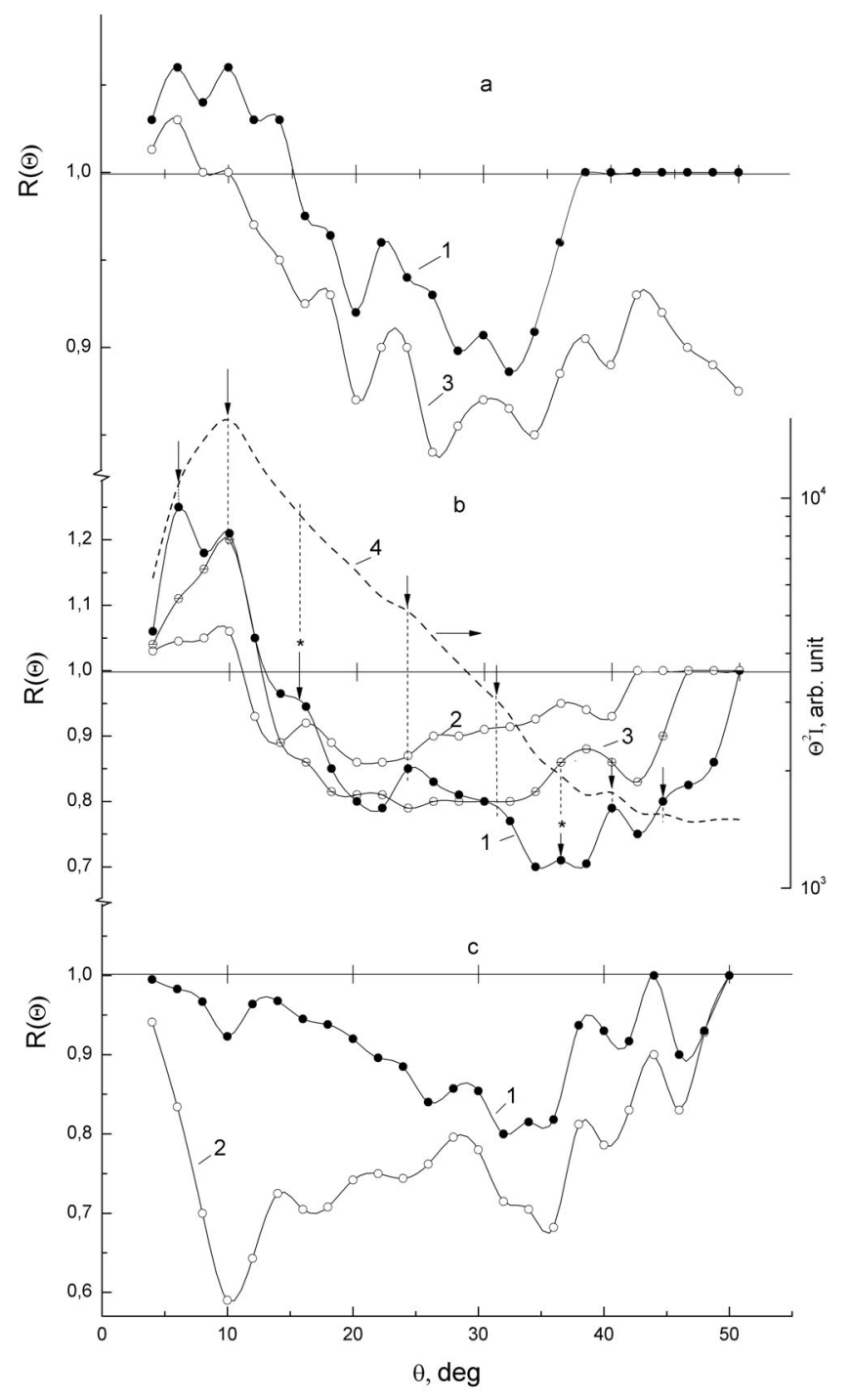

Fig. 2. Relative scattering indicatrices $R(\Theta)$ for the water with "typical" set of clusters $(a, b)$ and the water containing super-large clusters in its initial state (c) at different exposure times $t_{\mathrm{a}}$ and static magnetic field strengths $\vec{H}$ :

(1) $t_{\mathrm{B}}=10 \mathrm{~min}$, (2) $t_{\mathrm{B}}=30 \mathrm{~min}$, (3) $t_{\mathrm{B}}=60 \mathrm{~min}$; (a) $\vec{H}=1.44 \times 10^{4} \mathrm{~A} / \mathrm{m}$,

(b) $\vec{H}=5.84 \times 10^{4} \mathrm{~A} / \mathrm{m}$, (c) $\vec{H}=3.84 \times 10^{4} \mathrm{~A} / \mathrm{m}$. Curve 4 in figure $\mathrm{b}$ is calculated according to Eq. (1) at $t_{\mathrm{a}}=10 \mathrm{~min}$ and $\vec{H}=5.84 \times 10^{4} \mathrm{~A} / \mathrm{m}$. Vertical arrows indicate positions of maxima on curves 1 and 4 . 
$R(\Theta)>1$ due to increasing concentration of the large clusters with $r>0.9 \mu \mathrm{m}$, in the angular region defined as $15^{\circ}<\Theta \leq 40^{\circ}$ the inequality $\mathrm{R}(\Theta)<1$ holds true as a result of decreasing concentration of the small and medium-sized clusters (the sizes within the interval $0.24 \leq r \leq 0.63 \mu \mathrm{m}$ ), while in the region $40^{\circ} \leq \Theta \leq 50^{\circ}$ we have $R(\Theta)=1$ since there is no field effect on the small clusters $(r<0.24 \mu \mathrm{m})$. The increase in $t_{\mathrm{a}}$ leads to decreasing concentration of the initial clusters of all sizes and to a selective field influence, which is confirmed by decreasing $R(\Theta)$ in all the angular interval under study (see Fig. 2a, curve 3).

In addition, the effect of the field under these conditions stipulates forming of superlarge clusters with $\mathrm{r} \approx 6.7 \mu \mathrm{m}$ at $\mathrm{t}_{\mathrm{a}}=10 \mathrm{~min}$, with a gradual decrease of their sizes down to $\mathrm{r} \approx 3.3 \div 4.0 \mu \mathrm{m}$ when the exposure time increases up to $60 \mathrm{~min}$. This is testified by the fluctuations of $R(\Theta)$ observed in the angular region $\Theta>20^{\circ}$ (the period $\Delta \Theta \approx 4^{\circ}$ at $t_{\mathrm{a}}=10 \mathrm{~min}$ ), which have been absent in the $I(\Theta)_{\mathrm{b}}$ function. The period increases till $\Delta \Theta \approx 7^{\circ} \div 8^{\circ}$ in case of $t_{\mathrm{a}}=60 \mathrm{~min}$ (see Fig. 2a).

The increase of the field strength up to $5.84 \times 10^{4} \mathrm{~A} / \mathrm{m}$ at $t_{\mathrm{a}}=10$ min induces changes in the concentrations of large and medium clusters, similar to that observed at smaller $\vec{H}$ magnitudes and the same time of exposure, though in a more pronounced manner. Moreover, we have a decrease in the concentration of small clusters with $r<0.27 \mu \mathrm{m}$ (see curve 1 in Fig. $2 \mathrm{~b}$ ). Then the increasing time $t_{\mathrm{a}}$ gives rise to destruction of super-large clusters formed in the initial period of field action and a nontrivial change in the concentrations of large, middle and small clusters. So, the concentration of large clusters (in the region $\Theta>15^{\circ}$ ) at $t_{\mathrm{a}}=30 \mathrm{~min}$ passes through a minimum, always remaining greater than the initial one, whereas the concentration of middle and small clusters (in the region $\Theta \leq 15^{\circ}$ ) reaches a relative maximum, remaining less than the initial one (Fig. 2a, curves 2 and 3). Such a character of concentration changes evidences that, along with the processes of destruction of clusters, there are also processes of their formation. The character of changes in the concentrations of any clusters is determined by the relation of rates of these processes.

When the super-large clusters with $r \approx 6 \div 9 \mu \mathrm{m}$ are present in the initial water, the magnetic field leads to their destruction and also decreasing concentration of all the large, middle and small clusters. This is confirmed by the fact that the values $R(\Theta)<1$ are typical for the whole angular range of scattering * (Fig. 2c, curve 1). A characteristic feature of this process is prevailing decrease in the concentration of large clusters, taking place with increasing exposure time up to $30 \mathrm{~min}$ (Fig. 2c, curve 2). A further increase in $t_{\mathrm{a}}$ does not practically change the structural properties of water.

The mechanism for the influence of static magnetic field on the structural properties of water supposed by us consists in the following. According to [8], the water cluster represents a 3D net of coupled corrugated planes formed by hexagonal rings. Each of

\footnotetext{
* Periodic fluctuations of $R(\Theta)$ seen in Fig. 2c for the angular interval of $\Theta>20^{\circ}$ are caused by fluctuations of intensity of the initial scattering indicatrix.
}

Ukr. J. Phys. Opt. 2010, V11, №1 
these rings is composed of 6 atoms of oxygen located in its corners and 12 atoms of hydrogen, a half of which is situated inside the ring plane and another half is near the oxygen atoms on both sides from the ring plane. The latter ones are bound with each other and associated into a plane by covalent-hydrogen bonds. The oxygen atoms of every ring possess magnetic moments $\vec{m}$ equal, up to the order of magnitude, to the Bohr magneton. Obviously, every corrugated plane and a cluster in the whole have respectively the total magnetic moments $\vec{P}=\sum_{j} \vec{m}_{j}$ and $\vec{M}=\sum_{n} \vec{P}_{n}$, where j means the number of rings in the plane and $\mathrm{n}$ the number of planes composing a given cluster.

The external field $\vec{H}$ causes appearance of torques $\vec{N}_{r}=[\vec{m}, \vec{H}], \vec{N}_{p}=[\vec{P}, \vec{H}]$ and $\vec{N}_{c}=[\vec{M}, \vec{H}]$. They act respectively on every hexagonal ring, plane and cluster, of which magnetic moments are not parallel to $\overrightarrow{\mathrm{H}}$, and align the latter along the $\overrightarrow{\mathrm{H}}$ direction:

$$
\begin{aligned}
& A_{r}=\sum_{L}\left[\vec{m}_{L}, \vec{H}\right] \Delta \alpha_{L}=\sum_{L}\left[\vec{m}_{L}, \vec{H}\right] \omega_{L} t_{\theta}, \\
& A_{p}=\sum_{S}\left[\vec{P}_{S}, \vec{H}\right] \Delta \beta_{S}=\sum_{S}\left[\vec{P}_{S}, \vec{H}\right] \omega_{S} t_{\theta}, \\
& A_{c}=\sum_{i} \sum_{K=1}^{N_{i}}\left[\vec{M}_{K}^{i}, \vec{H}\right] \Delta \gamma_{K}^{i} V=\sum_{i} \sum_{K=1}^{N_{i}}\left[\vec{M}_{K}^{i}, \vec{H}\right] \omega_{K}^{i} t_{\theta} V .
\end{aligned}
$$

Here $L$ are $S$ denote respectively the numbers of hexagonal rings and the planes of scattering clusters of all types, $k$ the index indicating the number of clusters of $i$-type, $\Delta \alpha_{L}$, $\Delta \beta_{S}$ and $\Delta \gamma_{K}$ are respectively the rotating angles of the ring, the plane and the cluster, and $\omega_{L}, \omega_{S}$ and $\omega_{K}$ are the corresponding angular velocities describing the rotation.

The work of the magnetic field on the orientation of hexagonal rings (see Eq. (4)) and planes of clusters (see Eq. (5)) is related to breakage of the corresponding hydrogen bonds, thus resulting in destruction of clusters. It seems likely that the amount of energy required for complete orientation of the rings and planes depends on the initial angles between the neighbouring rings and planes of the cluster and those between the directions of $\vec{H}$ and $\vec{m}_{L}$ (or $\vec{P}_{n}$ ) vectors, as well as on the lengths of butt ribs of the planes. This energy decreases if the angles and lengths mentioned above do so.

It would be reasonable to suppose that in the small clusters having nearly spherical shapes, the planes forming the cluster are small, contain small number of hexagonal rings and their butt ribs are of small lengths. Then the curvature of external surface of such the clusters is the largest, which conditions small angles between the planes. Owing to all of these factors combined together, it costs the least for the external field to destruct the clusters, which is indeed observed in the experiments.

For the clusters of middle sizes, the curvature of the external surface decreases, thus resulting in increasing angles between the planes, squares of the planes, lengths of the butt ribs and, consequently, in increasing energy cost for the field to destruct the middle- 
sized clusters. That also conforms well to the experimental data available. Indeed, in the angular interval of $11^{\circ} \leq \Theta \leq 24^{\circ}$ that corresponds, in accordance with the classification [6], to the region of sizes of the middle clusters $(0.9 \geq \mathrm{r} \geq 0.4 \mu \mathrm{m})$, the $R(\Theta)$ values in Fig. 2a and Fig. 2b exceed those for the small clusters (see the interval $\Theta>24^{\circ}$ ) and, moreover, an increase in $R(\Theta)$ takes place with decreasing scattering angle in this interval. This substantiates that a destructing influence of the field diminishes with increasing sizes of the clusters.

Extrapolating the arguments given above to the case of large clusters, one can suppose that a more substantial, when compare to the middle clusters, increase in the interplane angles, the squares of the planes, the lengths of the butt ribs would provide sufficient solidity of construction of the large clusters, so that the energy of the magnetic fields used would be not enough to destruct these clusters. It should be noted that formation of inner planes can also increase solidity of the large clusters.

New large and super-large clusters are apparently formed from the remaining ones and from the fragments of middle and small clusters destroyed earlier, which have been preliminarily oriented along the field. Correlation of decreasing degree of the relative indicatrix $R(\Theta)$ observed in the angular interval of $\Theta \geq 16^{\circ}$ and its increasing degree in the small-angle area (Fig. 2a and Fig. 2b) testifies to this fact. New middle- and small-sized clusters are probably formed on the basis of fragments of destroyed clusters, which have an ordered magnetic orientation. Both the increase of the relative indicatrix seen in the angular interval of $\Theta \geq 16^{\circ}$ and its narrowing occurring at smaller angles with growing $t_{\mathrm{a}}$ time (see Fig. $2 \mathrm{~b}$ ) point to the process of formation mentioned above. As specified above, the simultaneous destruction process causes non-monotonic $R(\Theta)$ changes with the time $t_{\mathrm{a}}$.

Destruction of the large and super-large clusters due to the action of static field, in case if the latter are present in the initial water (see Fig. 2c), is presumably linked with the field work $A_{c}$ on their orientation in the direction of the $\vec{H}$ vector. Due to decrease of intercluster distance in the presence of super-large clusters, a major contribution to their destruction is presumably caused by counteraction of the friction force among them and among the clusters and intercluster environment. The friction force of any cluster is proportional to the friction coefficient $\mathrm{h}$ describing the action of liquid fraction of water (consisting of water molecules not bound in clusters) and that of the other clusters $(\xi)$. This force is also proportional to the square of the external surface $\left(S=4 \pi r^{2}\right)$, the linear velocity of movement of the cluster's surface with respect to the liquid phase or the other cluster $(v=\omega r)$, and the number of clusters $\mathrm{N}^{`}$ adjacent to that under consideration. This could be described as follows:

$$
F_{f r}=4 \pi h r^{3} \omega+4 \pi \xi r^{3} \omega N^{`}
$$

The work of the friction force is equal to product of that force and the average length of displacement of the surface of cluster rotated around the rotation axis till the directions of the magnetic moment $\vec{M}$ and the field strength $\vec{H}$ coincide. Any point of the cluster 
surface lying in the plane perpendicular to its rotation axis and passing through the cluster's centre, draws the largest arc $l$ equal to half its circle length, i.e. $l=\pi r$ in case of the opposite $\vec{M}$ and $\vec{H}$ directions. For the points of surface, through which the rotation axis passes, we have $l=0$. Therefore the average displacement length for any cluster surface point may be accepted to be equal to $\bar{l}=\pi r / 2$, if the cluster is rotated by the maximal angle. Super-large and large clusters are destructed when the field energy given by Eq. (6) exceeds the work of the friction force:

$$
\sum_{q} \sum_{K=1}^{N_{q}}\left[\vec{M}_{K}^{q}, \vec{H}\right] \omega_{K}^{q} t_{s} V>\sum_{q} \sum_{K=1}^{N_{q}} 2 \pi^{2}\left(r_{K}^{q}\right)^{4} \omega_{K}^{q} V\left(h+\xi N_{K}^{q}\right) .
$$

Here $q$ is the number of type of the large and super-large clusters $(q<i)$. It is the component of the friction force acting among the clusters that is likely to contribute mainly to destruction of these clusters, since the friction force appearing among the large clusters and the intercluster environment does not result in the values of $R(\Theta)<1$ for the samples with the "typical" set of clusters in the angular interval $\Theta<15^{\circ}$ (see Fig. 2a and Fig. 2b).

Decrease in the concentration of middle and small clusters in this case is obviously conditioned by the same reasons as in the water with a "typical" set of clusters, i.e. by the work of the field (see Eqs. (4) and (5)) on the orientation of hexagonal rings and planes of these clusters. Insignificant contribution of the field work to destruction of clusters during their rotation (see Eq. (6)) is confirmed by approximate equality of the $R(\Theta)$ values in a relevant angular interval of scattering for the water tests containing super-large clusters and a "typical" set of clusters (Fig. 2b and Fig. 2c). Presumably, the role of the friction force in destructing the middle and small clusters is insignificant since, due to their small sizes, during rotation imposed by the field they mainly suffer only the friction related to intercluster environment, which is not enough for their destruction.

Hence, it follows from the results presented above that the static magnetic field diminishes the total concentration of clusters, which could be interpreted as some destructurisation of water. However the character of its action on water depends on the initial set of clusters. So, in the presence of super-large clusters in the initial water, the field gives rise to their destruction and decreasing concentration of clusters of all the other sizes, mainly the large-sized ones. As a result, a more fine-dispersed structure is being formed, when compare to the initial one. If the super-large clusters are absent in the initial water, the magnetic field causes decrease in the concentration of middle and small clusters, increasing the concentration of large ones and forming super-large clusters. Eventually, this is concluded by forming a more rough-dispersed structure, when compare to the initial one.

\subsection{Light scattering in moving magnetic field}

We have revealed that the nature of influence of the static magnetic field rotating in a horizontal plane on the structural properties of water depends on such factors as the velocity $\omega$ and the mode of rotation, the set and concentration of clusters in the initial water, 
Table 1. Influence of rotated magnetic field on the parameters of light scattering by water with the "typical" set of clusters.

\begin{tabular}{|l|c|l|l|}
\hline \multicolumn{1}{|c|}{ State of water } & $\vec{H}, 10^{4} \mathrm{~A} / \mathrm{m}$ & $I_{\Sigma}$, per cent & \multicolumn{1}{c|}{$\mathrm{r}, \mu \mathrm{m}$} \\
\hline Initial & 0 & 100 & $1.05 ; 0.59 ; 0.46 ; 0.31 ; 0.22$ \\
\hline $\begin{array}{l}\text { After 3 revolutions } \\
\text { of the field }\end{array}$ & 1.44 & 96.5 & $4.5 \div 6.7 ; 1.09 ; 0.45 ; 0.31 ; 0.21$ \\
\hline $\begin{array}{l}\text { After 10 revolutions } \\
\text { of the field }\end{array}$ & 1.44 & 96 & $3.3 \div 4.0 ; 1.09 ; 0.47 ; 0.32 ; 0.23$ \\
\hline \hline Initial & 0 & 100 & $1.58 ; 0.83 ; 0.53 ; 0.34 ; 0.23$ \\
\hline $\begin{array}{l}\text { After 3 revolutions } \\
\text { of the field }\end{array}$ & 5.84 & 90.7 & $1.58 ; 0.95 ; 0.53 ; 0.32 ; 0.20$ \\
\hline $\begin{array}{l}\text { After 10 revolutions } \\
\text { of the field }\end{array}$ & 5.84 & 83.7 & $1.58 ; 1.0 ; 0.53 ; 0.32$ \\
\hline
\end{tabular}

the number $g$ of revolutions and the magnetic field strength $\vec{H}$. If $\vec{H} \leq 2.4 \times 10^{4} \mathrm{~A} / \mathrm{m}$, the influence of the field rotating with the velocity of $\omega_{0}=3 \div 4 \mathrm{rpm}$ on the water with the "typical" set of clusters has a selective nature: the minimal value of the relative indicatrix $R(\Theta)<1$ is observed in the small-angle region $10^{\circ} \leq \Theta<20^{\circ}$ after 3 revolutions of the field. This points out a predominant decrease in the concentration of medium clusters. A presence in the angular interval $20^{\circ}<\Theta \leq 45^{\circ}$ of fluctuations of scattered light intensity with the period $\Delta \Theta=4^{\circ} \div 6^{\circ}$, which has been absent in the indicatrices for the initial samples, testifies a formation of super-large clusters with $r \approx 4.5 \div 6.7 \mu \mathrm{m}$ (see curve 1 in Fig. 3). Increasing number of revolutions (up to $10 \mathrm{rpm}$ ) results in decrease of the size of these clusters down to $r=3.3 \div 4.0 \mu \mathrm{m}$, decrease in the concentration of large clusters and recovery of concentration of the smallest clusters $(r<0.25 \mu \mathrm{m})$ up to the initial concentration.

Increase in the magnetic field in the region of $\vec{H}>2.4 \times 10^{4} \mathrm{~A} / \mathrm{m}$ imposes a decrease of selectivity of its influence on the medium and small clusters, a more substantial decrease of their concentration, a slight increase of the concentration of large clusters $\left(\mathrm{R}(\Theta)>1\right.$ in the angular region of $\left.\Theta<10^{\circ}\right)$, and formation of super-large clusters with $r \approx 4.5 \div 5.5 \mu \mathrm{m}$ after the first two or three revolutions of the field (see Fig. 3, curve 2). Increase in the $\mathrm{g}$ parameter up to $10 \mathrm{rpm}$ causes decrease of their radius down to $r \approx 3.5 \mu \mathrm{m}$.

Besides of the changes of structural properties mentioned above, there are also slight changes in the sizes of some of the large, medium and small clusters, disappearance of particular medium and small clusters, as well as decrease in the integral scattered intensity, the latter effect being increased for larger $\vec{H}$ and $g$ values (see Table 1).

When the super-large clusters with $r \approx 4.5 \div 6.7 \mu \mathrm{m}$ are present in the initial water, rotation of the magnetic field with the velocity of $3 \div 4 \mathrm{rpm}$ leads to their destruction and increase in the concentration of medium and small clusters, the process being accumulated substantially with increasing number of revolutions. That is confirmed by the value $R(\Theta)>1$ inherent in the angular interval of $\Theta>10^{\circ}$ (see Fig. 3b, curves 3 and 4). 
After $2 \div 3$ revolutions the field causes significant decrease of the cluster concentration in the water containing higher concentrations of medium and small clusters in its initial state. The increase in the g parameter does not produce any further noticeable changes in the structural properties (see Fig. 3c).
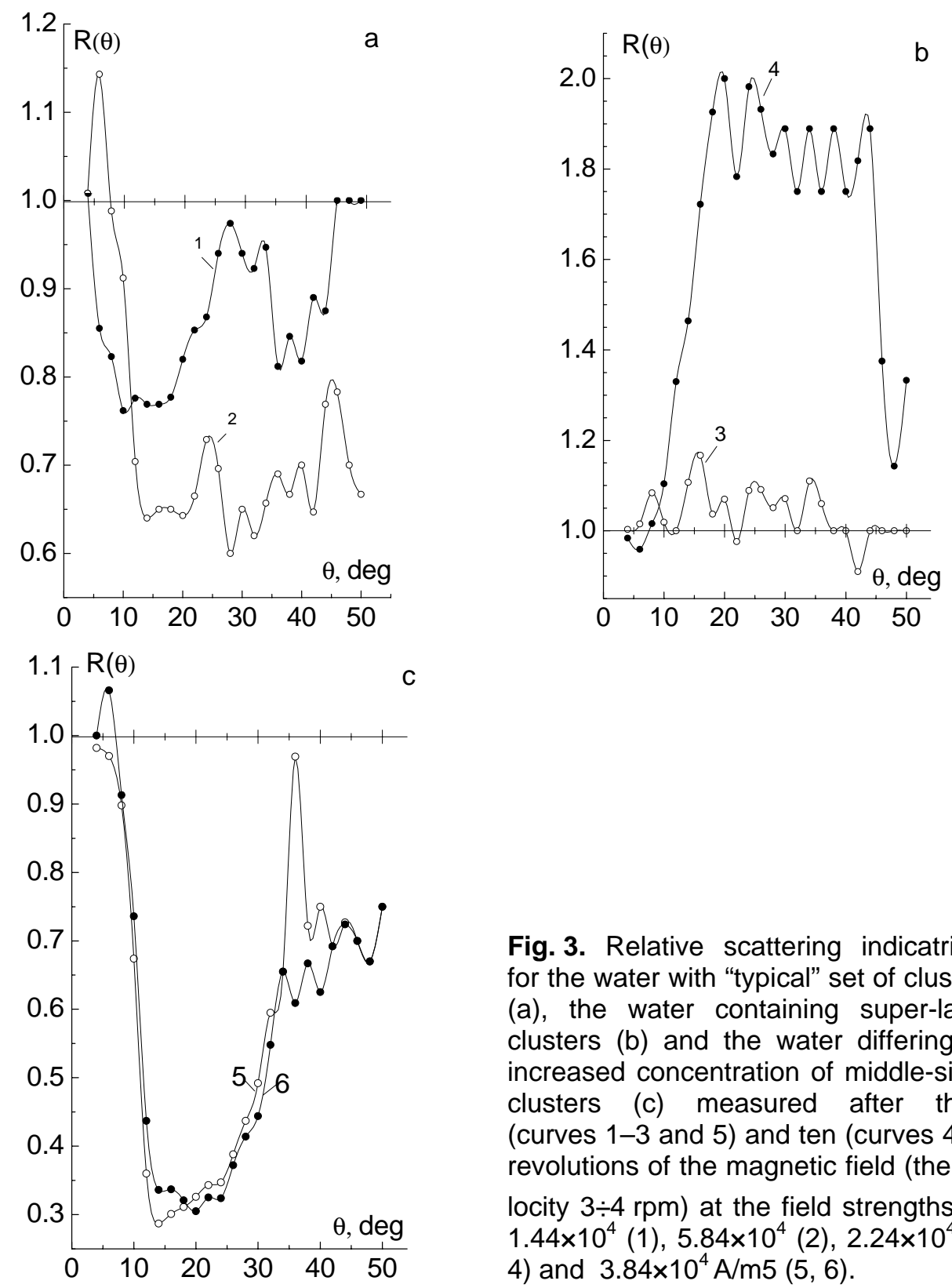

Fig. 3. Relative scattering indicatrices for the water with "typical" set of clusters (a), the water containing super-large clusters (b) and the water differing by increased concentration of middle-sized clusters (c) measured after three (curves 1-3 and 5) and ten (curves 4, 6) revolutions of the magnetic field (the velocity $3 \div 4 \mathrm{rpm}$ ) at the field strengths $\overrightarrow{\mathrm{H}}$ $1.44 \times 10^{4}(1), 5.84 \times 10^{4}(2), 2.24 \times 10^{4}(3$, 4) and $3.84 \times 10^{4} \mathrm{~A} / \mathrm{m} 5(5,6)$.

The increase in the velocity of field rotation up to $\omega=10 \div 12 \mathrm{rpm}$ does not change the nature of field influence on the structural properties, as compared to the case of $\omega_{0}=3 \div 4 \mathrm{rpm}$. However, it yields in decrease of its degree by $50 \div 70 \%$. The nature of influence of the field changes for the case of $\omega<\omega_{0}$, in comparison with that observed at $\omega_{0}$. From Fig. 4 (curve 1) it is clear that after one revolution of the field 
$(\omega \approx 1 \div 1.5 \mathrm{rpm})$ there is a slight decrease in the concentration of large clusters and a predominant increase in the concentration of medium clusters (the same concerns the small ones, though in a less degree). All of these factors produce increase in the integral intensity $I_{\Sigma}$ of scattering by $\sim 10 \%$.

In the same Fig. 4 (curve 2) we have shown the relative indicatrix observed after discrete revolution of the field with the steps of $15^{\circ}$ and the stops for $5 \div 10 \mathrm{~s}$ occurring after every step. It is obvious that this method of influence imposes some decrease in the concentration of large clusters, as well as practically uniform and slight increase in the concentration of both medium and small clusters and in the $I_{\Sigma}$ value (by $4 \div 5 \%$ ). A peculiarity

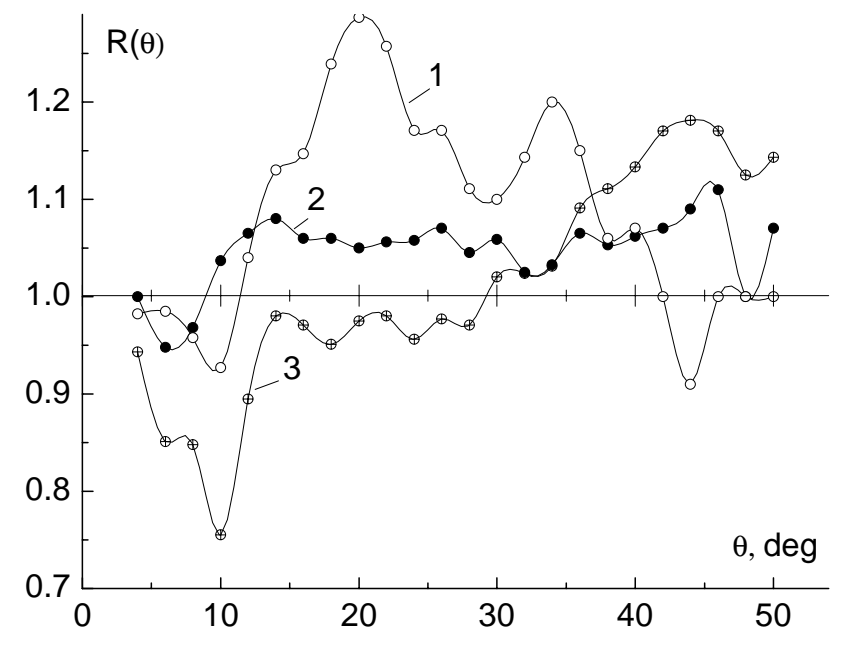

Fig. 4. Relative scattering indicatrices for the water with "typical" set of clusters after slow continuous $(\approx 1 \mathrm{rpm}$, curve 1 ) and discrete (curve 2) revolutions and after repeated reversals of the magnetic field (curve 3 ). $\vec{H}=5.84 \times 10^{4} \mathrm{~A} / \mathrm{m}$.

of discrete field rotation lies in the least degree of its influence on the structural properties $(0.95 \leq R(\Theta) \leq 1.05)$, when compare to the other modes of influence mentioned above.

Cyclic reversal of magnetic field direction with the rate of 12 cycles per minute during 4 min (without any rotation) diminishes mainly the concentration of large and (in a less degree) medium clusters and, at the same time, gives rise to approximately the same increase in the concentration of small clusters (see curve 3 in Fig. 4).

The effect of the rotating magnetic field on the structural properties of water originates from the same factors as for the case of the static field. However, while the field rotates, its work on aligning of the components of clusters and the clusters as a whole is equal:

$$
A_{r}=\sum_{L}\left[\vec{m}_{L}, \vec{H}\right] 2 \pi g, \quad A_{p}=\sum_{S}\left[\vec{P}_{S}, \vec{H}\right] 2 \pi g, \quad A_{c}=\sum_{i} \sum_{K=1}^{N_{i}}\left[\vec{M}_{K}^{i}, \vec{H}\right] 2 \pi g V,
$$

is considerably greater due to large rotating angles $\Delta \alpha=\Delta \beta=\Delta \gamma=2 \pi g$. This evokes more essential changes in the concentration and size of the clusters of different types. An additional factor is also worth noticing which enhances the influence of rotating field on 
the structural properties of water. Namely, the orientation effect of that field concerns, with no exception, to all "construction" elements of clusters and clusters themselves. On the contrary, in case of the static magnetic field some of hexagonal rings, planes and clusters, whose magnetic moments have initially coincided with the field direction, are not being affected by the field.

Different natures of selectivity of the effect of weak static and rotating fields on the structural properties of water consist presumably in the following. As already mentioned, small clusters are destroyed by the static magnetic field in the first place, mainly because of breaking bonds among the hexagonal rings and planes during their orientation along the field. Rotation of such clusters along the $\vec{H}$ direction takes place practically without resistance of intercluster environment, due to their small sizes and, consequently, a small square of intercluster boundaries.

When the field rotates, the influence of factors $A_{r}$ and $A_{p}$ involved in Eqs. (9) grows because of larger rotation angles. In addition, the square of cluster's surface and the square of intercluster boundaries increase with increasing cluster size, while the intercluster distances then decrease. Therefore the influence of friction force among the clusters during their orientation associated with the energy $A_{c}$ (see Eqs. (9)) becomes determining in destruction of the medium-sized clusters. This is especially important in the case of high concentration of the latter in the initial water (Fig. 3c). An increase in the field strength $\overrightarrow{\mathrm{H}}$ enhances the effect of factors $A_{r}$ and $A_{p}$ in Eqs. (9), resulting in weakening selectivity of the field influence.

Super-large and large clusters are obviously formed from the fragments of medium and small clusters, just like for the case of static field. However, the orientation energy $A_{c}$ given by Eqs. (9) for the case of rotating field is much larger than that for the static filed (see the relation for $A_{c}$ given by Eq. (6)), which provides higher ordering of magnetic orientation of the fragments and makes possible formation of the mentioned clusters in the process of "intermixing" of polydisperse water environment after the first revolutions of the field. It might be supposed that increasing number of field revolutions would result in smoothing out irregularities, "roughness" and structural defects of the cluster surfaces by the friction force, which then would cause approaching of their shapes to a spherical one and could lead to some decrease in their sizes.

As in the case of static field, destruction of super-large clusters contained in the initial water under the action of rotating field is evidently caused by decrease in the intercluster distances and by action of the friction force among these clusters appearing in the process of their orientation along the field direction.

Different effects of the velocity and the mode of rotation of the field on the structural properties of water are probably associated with dependence of forces of cluster reaction on these rotation parameters. So, an increase in the velocity of rotation of the field generates the inertial force, which leads to decrease in the degree of destruction of clusters. Slow continuous rotation increases probabilities for bonding of separate rings into a plane, bonding of planes among themselves or bonding of individual fragments of clus- 
ters, thus assisting formation of new clusters. When the field rotates discretely, elastic forces of the clusters preserve, to a large extent, the initial spatial configuration, concentration and sizes of clusters, weakening substantially the action of friction force directed at any changes in the structural properties.

Abrupt changes in the direction of friction forces and the action of inertia happening with repeated reversals of the field result in destruction of large and medium clusters, the fragments of which increase the concentration of small clusters. The latter are affected substantially less by these forces, due to a presence of free intercluster space among most of them and due to their small mass.

Thus, the effect of the magnetic field on the structural properties of water in case of rotation of the former with the velocity of $3 \div 4 \mathrm{rpm}$ depends on the initial structural state of water. It is similar to that occurred in case of the static field, though more considerable. Unlike the latter case, rotation of the field stipulates insignificant changes of clusters' sizes in the water with "typical" set of clusters, as well as a tendency to decreasing polydispersity. In the water containing super-large clusters, the field rotation destroys them and forms middle and small clusters, resulting in a considerable increase of total concentration of clusters, when compare to the initial one.

The changes in the parameters of field rotation (i.e., the number of revolutions and the rotation velocity), use of the discrete rotation or cyclic reversal of the field affect the basic characteristics of the structural properties such as the total concentration of clusters, the degree of polydispersity and its dimension (low or high dispersity). These effects could be employed for controlled changes of the structural properties of water.

\section{Conclusion}

The data presented here point out a significant influence of static magnetic field on the structural properties of water. This is revealed in the apparent changes of concentration and set of clusters with different sizes. A degree and character of these changes depend upon the mode of the field (stationary or moving one), its strength and the time of exposure, the initial structure of water, and the parameters of field rotation.

The changes in the structural properties of water are associated with the work of field on aligning, along the field direction, magnetic moments of the clusters' elements and the clusters as a whole. This work is needed in order to break the hydrogen bonds among the elements of clusters and overcome the friction force appearing among clusters during their rotation and resulting in their destruction. On the other hand, the filed forms new clusters with ordered magnetic orientation from the fragments of those clusters which have been destroyed or still remained unharmed.

\section{References}

1. Klassen V I, Water and magnet. Moscow: Nauka (1973). p.111.

2. Kulskiy L A, Dal' V V and Lenchina L G, Well-known and unknown water. Kyiv: Radyanska shkola (1982). p.120. 
3. http://provodu.kiev.ua/oleg-mosin

4. http://www.bestwater.ru/page/mbckema1.html

5. http://andeg.com.ua/site/page26483.html

6. Kovalenko V F, Levchenko P G, Shutov S V and Bordiuk A Yu, 2009. Investigation of the nature of light scattering by water. Ukr. J. Phys. Opt. 10: 38-53.

7. Kovalenko V F, Shutov S V and Bordiuk A Yu, 2009. Interferentional effects in light scattering of biological liquds. Biomedical Radioelectronics. 8: 72-78.

8. Sinitsin N I, Petrosyan V I, Yolkin V A, Devyatkov N D, Gulyaev Y V and Betskij O V, 1999. A special role of the system "millimeter waves - water environment" in the nature. Biomedical Radioelectronics. 1: 3-21.

Kovalenko V.F., Bordyuk A.Yu. and Shutov S.V., 2010. Light scattering of water under magnetic field. Ukr.J.Phys.Opt. 11: 6-20.

Анотація. При $T=300$ К досліджено вплив постійного магнітного поля напруженістю $\vec{H} \leq 6,4 \times 10^{4} \mathrm{~A} / \mathrm{M}$ на структурні властивості води на основі вимірювання кутових залежностей інтенсивності розсіяного лазерного випромінювання. Цей вилив проявлявся y зміні основних характеристик структурності: концентраиії $i$ набору кластерів, їх розмірів. Ступінь і характер иієї зміни залежали від способу впливу поля (нерухоме та обертове), його напруженості $i$ часу впливу, набору $і$ концентраиії кластерів у вихідній воді, параметрів обертання поля. Зміна структурності води обумовлена роботою поля щодо орієнтаиії магнітних моментів елементів (гексагональних кілеиь, площин) кластерів і самих кластерів у напрямку дії поля, що витрачається, з одного боку, на розрив водневих зв'язків між елементами кластерів, а також на подолання сили тертя кластерів при ïx розвороті в магнітному полі, $i$, з іншого боку - на формування нових кластерів 3 фрагментів зруйнованих і цілих кластерів, але з упорядкованою магнітною орієнтаиією. 